\title{
The contributions of anatomical studies to knowledge of perceptual processing
}

\author{
Ray W. Guillery \\ Department of Anatomy Marmara University Haydarpaşa Istanbul, Turkey
}

\begin{abstract}
In many contemporary studies and textbooks perceptual processing is treated as a 'pure sensory' phenomenon, one that can be understood on the basis of pathways passing information from the sensory periphery to the cerebral cortex, for processing within the cortex and subsequent passage to motor centers or memory stores. However, many physiologists, psychologists and philosophers have recognized perceptual processing as closely dependent upon action (e.g. the 'sensorimotor contingencies' of O'Regan and Noë, 2001)', although the anatomical nature of the functional links is generally left unresolved. A survey of pathways that pass messages through the thalamus to the cerebral cortex (visual, tactile etc.) shows that these are not 'pure sensory' pathways. They are generally branching axons that convey messages to lower, motor centers with one branch and to the thalamus with the other. That is, since the two branches will be transmitting the same message, the thalamic relay receives information not only about sensory events but also about concurrent instructions that are on the way to motor centers. This dual information, about sensory events and motor instructions, is an implicit part of the message that the thalamus passes to cortex. The axonal branching patterns reveal an anatomical basis of sensorimotor contingencies, which cortical mechanisms are not likely to ignore even when experimental studies do not reveal them.
\end{abstract}

Key words: thalamus; cerebral cortex; sensory mechanisms; sensorimotor events; cortical outputs

Anatomy 2008; 2: 9-15, (c) 2008 TSACA

\section{Introduction}

\section{The 'classical' view of sensory pathways}

"Visual perception can be... understood as the activity of exploring the environment in ways mediated by knowledge of the relevant sensorimotor contingencies."

The sensory pathways of the brain were largely defined during the second part of the 19th century by clinicians and anatomists. The basic evidence, from studies of clinical lesions and of normal and degenerating central pathways, showed somatosensory, visual and auditory pathways passing to the thalamus, and thence to the cerebral cortex. Each pathway involves a distinct region (nucleus) of the thalamus and passes to a specific cortical area. Modern textbooks present these well- established pathways as well as pathways, for gustatory or vestibular afferents, which were defined later. Other ascending afferents to the thalamus come from the cerebellum or the mamillary bodies, and each, again, relates to a particular thalamic nucleus and a specific cortical area. Essentially all of the messages that reach the cerebral cortex from peripheral receptors must first pass through the thalamus. Only olfactory inputs reach the cerebral cortex without first passing through the thalamus. Further central processing as defined by contemporary studies is thought to occur in a series of parallel and hierarchical corticocortical connections that eventually lead to memory or to motor outputs (Figure 1). ${ }^{2-3}$

This anatomical view of thalamocortical relationships, schematically represented by the interrupted lines 
in Figure 1, dominates current views of sensory processing and has been greatly enriched by experimental studies that define how the activity of nerve cells in each sensory pathway relates to external events. For example, there is now a great deal of evidence for the visual pathways about the nature of neural responses to sensory stimuli that reach the visual cortex (area 17), ${ }^{4}$ and how these are further 'processed' within the cortical areas concerned with visual functions. ${ }^{5-8}$ Similarly, auditory and somatosensory pathways are often viewed primarily in terms of their corticocortical connections.' Sensory processing, in this 'classical' view, involves activity in prethalamic nerve cells, which is passed to thalamic nerve cells and thence to the cerebral cortex. Some sensory processing occurs at prethalamic levels, but the thalamic relay itself appears to pass messages to cortex that hardly differ from those that the thalamus receives, and further sensory processing is then thought to occur primarily or entirely in the hierarchically interconnected areas of the cerebral cortex.

This sketch of the classical view of thalamocortical organization appears to demonstrate how events in the external world can generate neural activity that is processed through thalamic and cortical relays to produce a perceptual outcome, and the observations I have summarized are commonly presented in this way (interrupted lines in Figure 1). In this essay some of the problems that remain unresolved for this model will be raised, and the anatomical problems will form a main part of my argument. However, the anatomical problems do not stand in isolation. They relate closely to behavioral and philosophical problems about the nature of perceptual processing that have been raised in the past, which show the limitations of the classical view of sensory mechanisms.

\section{Some of the Problems that Remain}

\section{Large parts of the thalamus are not accounted for in the classical view}

More than half of the primate thalamus is made up of thalamic nuclei that are not adequately dealt with in the above account. These include large thalamic nuclei such as the pulvinar, the lateral posterior or lateral dorsal nuclei that send axons to parietal and temporal cortex, the mediodorsal nucleus and significant parts of the intralaminar complex that have axons going to frontal cortex. The contributions of these thalamic cell groups to cortical processing are largely ignored by most current accounts of cortical function, or are treated as providing modulatory inputs to the ongoing cortico-cortical circuitry. ${ }^{10-11}$ However, there is significant evidence that many of these cell groups can play a role in transmitting messages from one cortical area to another through the thalamus, ${ }^{12-13}$ and they have been called higher order relays (HO in Figure 1) for that reason. The way in which the functions of the direct corticocortical connections relate to those of the higher order cortico-thalamocortical connections (see e.g. ${ }^{6,14}$ ) are currently unknown and merit experimental study.

In so far as it is reasonable to consider that all parts of the neocortex have a common pattern of organization, ${ }^{15-17}$ it is necessary to recognize that for the areas of cortex that have been most closely studied, that is for the primary sensory areas, the functional inputs that play a primary role in information processing within those cortical areas come from the thalamus. On this basis it is reasonable to believe, until evidence to the contrary is available, that the same holds for all cortical areas, including those receiving higher order inputs. That is, in order to understand higher cortical areas it may be profitable to look for the functional properties of their thalamic inputs, which may reveal more than the very limited information that is currently available about the functions of the direct corticocortical inputs. Defining the functions of higher order thalamic relays such as the pulvinar or the mediodorsal nucleus should be treated as a high priority.

\section{Essentially all cortical areas have outputs to brain- stem or spinal centers that themselves have links to motor centers}

The major cortical outputs to subcortical structures come from cortical layers 5 and 6 . The layer 5 output is particularly important for demonstrating that most cortical areas themselves have a significant capacity to act 
Figure 1. Schematic representation of the major pathways discussed in the text. The interrupted lines show a highly simplified representation of the classical view and the continuous lines show a simplified representation of the pathways described in this essay. 1: This represents sensory inputs (visual, auditory, somatosensory etc.) as well as efferent pathways from the cerebellum and the mamillary bodies. 5: cells in cortical layer 5 that send corticofugal axons to the brainstem and spinal cord with branches going to the thalamus. FO: first order thalamic relays that receive afferents from (1) for relay to cortex. HO: higher order thalamic relays that receive afferents from layer 5 of cortex for relay to higher cortical areas. Patterns of impulse traffic are shown schematically for some of the branching axons as parallel vertical lines to indicate that generally the pattern of activity in the parent branch is passed into each of the daughter branches. The arrows with interrupted lines represent a simplified version of the classical view, showing the hierarchy of cortical connections, but not showing the parallel processing.

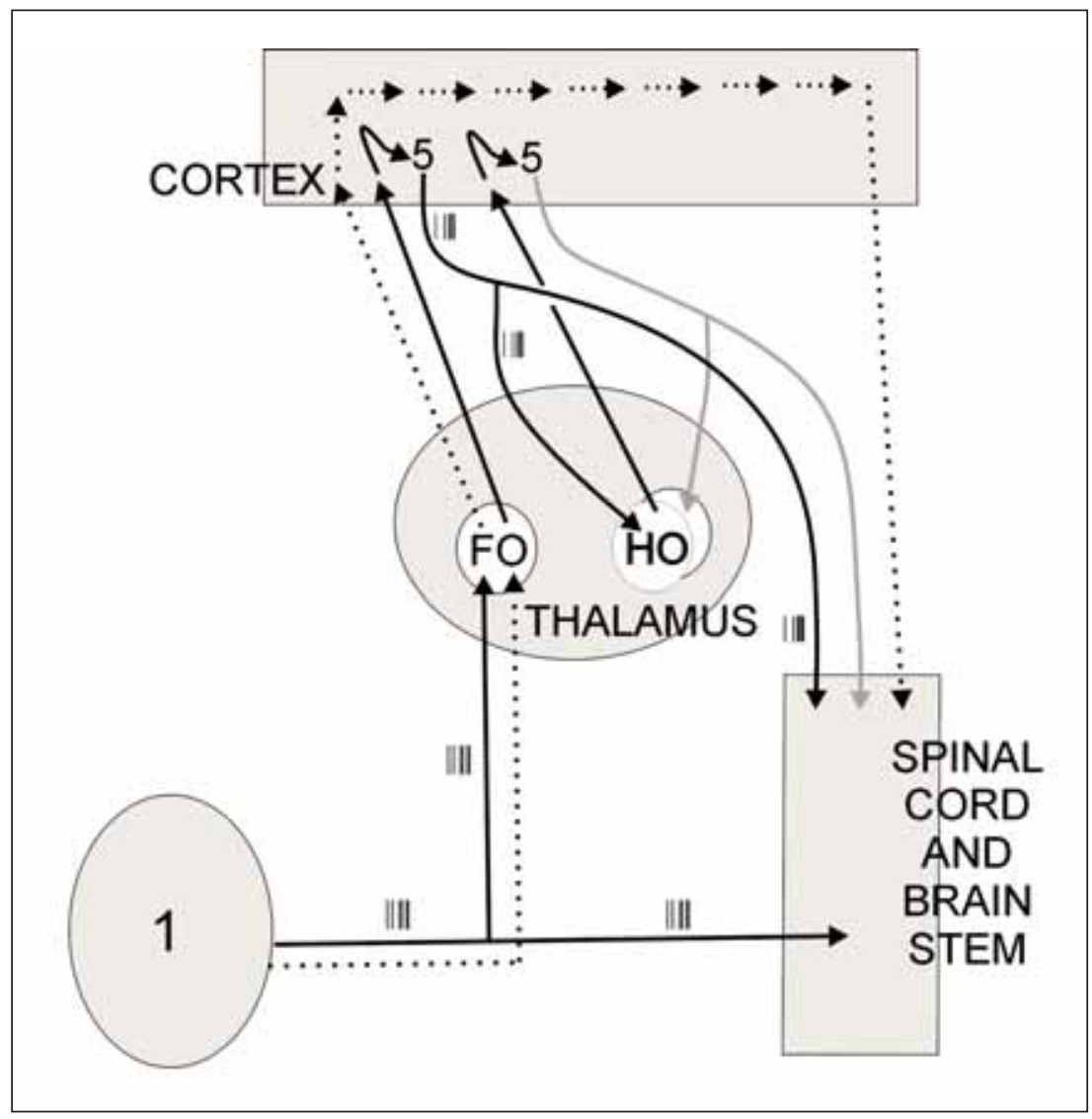

directly on subcortical structures, and that the actions of a cortical area cannot be judged only, or even primarily, on the basis of their corticocortical connections. Harting's study ${ }^{18}$ of 24 separate cortical areas in the cat, each with an output to the superior colliculus is a striking demonstration of the extent to which most cortical areas can gain access through lower centers to motor outputs, without necessarily involving any part of the hierarchy of corticocortical processing. If we wish to understand the functional role of a cortical area, then it is essential to know how it can act on subcortical centers. One powerful way of learning about the processing that occurs within any cortical area is to ask how that piece of cortex modifies the input it receives from the thalamus (or from other cortical areas, see section above) converting it to the output going to lower centers. Here the layer 5 output may prove to be the most revealing.

There are two reasons for focusing on the layer 5 outputs rather than the layer 6 outputs from cortex. One is that the layer 6 outputs appear to be limited to the telencephalon and diencephalon, whereas the layer 5 outputs go to brainstem and spinal cord as well. The second reason is the layer 6 inputs to the thalamus are modulatory; the properties of the layer 6 cells are not transmitted to cortex through the thalamus. From the point of view of the thalamic pathways, layer 6 is likely to prove important for understanding how cortex modulates its inputs, whereas layer 5 will show how cortex acts upon lower centers of the brain or upon other cortical areas.

The pathways that are afferent to the thalamus are generally made up of branches that come from axons having motor actions; understanding the functional role of these axons plays no part in the classical view

Many of the layer 5 outputs that go to brainstem or spinal cord have branches that provide a first link in the 
corticothalamocortical connections mentioned above for higher order relays (see above; Figure 1). Comparably, the ascending axons that reach the first order thalamic relays from sensory pathways, from the cerebellum or from the mamillary bodies (together shown as ' 1 ' in Figure 1), are branches of axons that innervate centers linked to motor actions (summarized in Guillery RW). ${ }^{19}$ That is, the axons that are afferent to the thalamus are mainly, perhaps entirely branches of axons that have a function distinguishable from that of the thalamocortical relay itself, and this function relates closely to action.

It is important to recognize that a branching axon generally sends the same message into each branch (Figure 1), producing a situation for the thalamocortical pathways that provides two distinct but related types of information to cortex. One is information about a currently active instruction about action on its way to motor centers, and the other concerns the nature of the activity in the peripheral receptors, or the nature of cerebellar, mamillary or cortical layer 5 activity. The pattern of branching shown in Figure 1, which characterizes most, possibly all of the pathways that carry information for relay to the cerebral cortex, allows us to see the thalamus as a monitor of motor outputs in the first place, and as a sensory relay secondarily. Contemporary views of thalamocortical functions have focused on the sensory relay and have ignored the monitor of motor outputs. The weakness of this approach can be understood by considering a general who receives a message about the movements of enemy forces and of the counteraction taken by his own forces, and who then takes note of the first part of the message but ignores the second part.

For the major sensory pathways and for the mamillary pathway going to the anterodorsal nucleus, concerned with head direction, ${ }^{20}$ we have good information about the nature of the sensory message reaching the thalamus, but we know little or nothing about the significance of the copy of the concurrent message on its way to the motor centers. That is we have no information about the action that the cortex is able to monitor through their thalamic inputs from these pathways. For the layer 5 inputs to thalamus, and for the majority of mamillothalamic and cerebellothalamic axons we know almost nothing about the nature of the messages traveling in either branch.

The implications for studies of cortical functions of these branching inputs to the thalamus are important, but far from simple. One important point is that the higher order thalamic relays can provide information through their thalamic relays to higher cortical areas about the ongoing motor instructions that are currently being issued by lower cortical areas. The hierarchies established by these connections have not been well defined. They merit detailed study so that they can be compared with the hierarchies formed by the direct corticocortical pathways 5-6 and seen in relation to developmental sequences. ${ }^{21}$

Defining the questions that should be asked about how the information that is on its way to motor centers can be read in the activity of cortical neurons receiving the thalamocortical inputs will prove to be a key issue for understanding the neural mechanisms that underlie the close relationship between action and perception (see, below). We need to understand the actions that are being monitored as well as the messages that are initiating these actions. This will not be simple. The difficulty of defining the functional properties of cortical cells was well illustrated by Hubel and Wiesel ${ }^{4}$ in their account of how they first found orientation responsive cortical cells: by accident when a new visual image was inserted into their slide projector. To understand the full significance of a sensory message reaching the cortex from the thalamus, we will need to know not only the sensory events that are encoded but also something about the ongoing motor instructions.

After raising this issue recently, I was accused, in a private conversation, of suggesting that experimentalists should be looking for functional clues in regions where (at present) there is no light, and where the information I am asking for cannot be found. This was a fair comment. Much of experimental cognitive neuroscience today is defining structures and functions in well illuminated regions, almost always of cortex. Younger investigators should be encouraged to recognize the impor- 
tance of illuminating new regions; this will be a more difficult, but in the long run far more rewarding endeavour, and may reveal keys that at present are lost to neuroscience.

\section{Many earlier investigators recognized that percep- tion is more closely related to action than can be represented by the classical view}

The idea that perception is related to action is not new, nor is the notion that branching axons in the nervous system can obliterate the distinction between the sensory and motor actions of an axon. There is a rich literature relating to these issues, and only a few examples can be considered here.

Two older, quite different, well recognized views that have related action to perception, and that can now be looked at in the light of Figure 1, are those of Helmholtz $^{22}$ on the one hand and of Merleau-Ponti ${ }^{23}$ on the other.

Helmholtz stressed the importance of action when he wrote:

"If we ask whether there exists some common characteristic distinguishable by direct sensation through which each perception related to objects in space is characterized for us, then we actually find such a characteristic in the circumstance that bodily movement places us in different spatial positions relative to the perceived objects, and in doing so also changes the impressions which these objects make on us. The impulse to movement, however, which we give through the innervation of our motor nerves is something which can be perceived directly. We feel we are doing something when we give such an impulse. But what it is we are doing we do not know directly."

It is interesting to notice the tension between the conscious and the unconscious aspects of the relationship that Helmholtz is describing, and to understand this in relation to the fact that first order thalamic relays (FO in Figure 1) are monitoring spinal and brainstem effects on motor actions, which will not be 'known directly', whereas many of the higher order thalamic relays will be monitoring cortical effects on motor actions, which may well be 'known'.

\section{Merleau-Ponti wrote: ${ }^{23}$}

"The object which presents itself to the gaze or the touch arouses a certain motor intention which aims not at the movements of one's own body, but at the thing itself from which they are, as it were, suspended."

This statement includes two key insights in just one sentence. One insight is the "certain motor intention". This need not produce a movement; it can lead to a movement, can fail to produce a movement because some other activity blocks it, or it can itself block a movement. The issue as to whether the "intention" is volitional or whether it simply implies activity directed towards the production of movement is left open. The second insight has the motor intention aimed not at the relevant parts of the body, at muscle groups or at groups of motor neurons, which would be the classical physiological approach to movement, but at the object that is being seen or felt, and from which the intended movements are "as it were, suspended". That is, the 'intention' relates to the object of vision or touch in an essentially mysterious way, which Merleau-Ponti encapsulates by having the movement suspended, but not quite suspended, rather, "as it were" suspended, from the object. This mystery is clearly unsatisfactory, but it is important, and for the neuroscientist it needs to be explored further from the point of view of the neural processes that may be represented by the statement. At least some of this mystery can be resolved by the connections shown in Figure 1, where the movement is related either directly to the object through the first order sensory pathways (FO), or indirectly through the outputs of a hierarchy of cortical connections represented by the higher order corticothalamocortical pathways (HO).

The puzzling relationship of action to perception has recently been discussed by Noë who has written critical accounts that stress the importance of understanding how action relates to perception and that demonstrate the limitations of the classical approach to perception. ${ }^{1,24-25}$ Churchland et al. had earlier written an account of vision showing that the classical approach, which they called the theory of 'pure vision', failed to account for many sensory phenomena. ${ }^{26}$ Berthoz recognized that branching 
axons could remove the distinction between sensory and motor pathways. ${ }^{27} \mathrm{He}$ wrote: 'The ... character of perception includes signals that derive not from the senses but from the intention to move.'

To some extent the literature on 'embodiment' relates to the issues raised here, but it is important to notice that embodiment often includes the motor reaction to a sensory stimulus, that is, how one can respond to it, and this needs to be distinguished from the specific issue raised in this essay, the direct motor involvement that is an intrinsic part of the sensory experience itself. ${ }^{28-29}$

\section{Conclusions}

The major contribution that this essay adds to earlier accounts of action and perception relates specifically to the branched nature of axons that carry messages to the thalamus for transfer to cortex. This pattern of branching shows the thalamocortical pathways serving as monitors of motor outputs. Essentially everything that reaches the cerebral cortex is a record of a sensorimotor event, an "enactment" between the organism and the environment. The cortex probably does not receive "pure" sensory inputs.

From an evolutionary point of view, the first thing a pathway from a sensory receptor needs in order to provide some selective advantage is a motor output. It is only when a motor link exists that the sensorimotor activity needs to be monitored so that the output can be modified by higher order connections or the activity in the sensorimotor link itself (not the input component alone) stored in memory for future reference. The anatomical evidence about branching patterns of functional inputs to first and higher order thalamocortical relays suggests that the thalamus can be regarded as providing information to cortex about sensorimotor links in the first place, and as pathways relating to memory storage and perceptual processing only secondarily. In so far as cortical cells represent anything, they represent sensorimotor links established at subcortical levels, and for cortical cells the sensory part of the link cannot be separated from the motor part. For any interpretation of cortical activity we have to look at the relation- ships that exist between stimuli delivered to sense organs and the reactions of the organism to those stimuli. Our cortical cells, by the very nature of their connections, are unable to separate sensory stimuli and what, if anything they represent in the 'real world', from our motor reactions and whatever those are telling us about our 'volitions'.

\section{References}

1. O'Regan JK, Noë A. A sensorimotor approach to vision and visual consciousness. Bebav Brain Sci 2001; 24: 939-73. ['sensorimotor contingencies' in this quotation refers to dependence of sensory and motor events upon each other.]

2. Kandel ER, Schwartz JH, Jessel TM. Principles of Neurocience. 4th ed. New York: McGraw-Hill; 2000.

3. Purves D, Augustine GJ, Fitzpatrick D, Katz L, LaMantia A-S, McNamara JO. Neuroscience. Sunderland, MA: Sinnauer Associates; 1997.

4. Hubel DH, Wiesel TN. Brain and visual perception. Oxford: Oxford University Press; 2005.

5. Van Essen DC, Anderson CH, Felleman DJ. Information processing the primate visual system: an integrated systems perspective. Science 1992; 255: 419-23.

6. Van Essen DC. Corticocortical and thalamocortical information flow in the primate visual system. Prog Brain Res 2005; 149: 17385 .

7. Shipp S, Zeki S. The organization of connections between areas V1 and V5 in macaque visual cortex. Eur 7 Neurosci 1989; 1: 30932.

8. Zeki S. A Vision of the Brain. Oxford: Blackwell; 1993.

9. Smiley JF, Hackett TA, Ulbert I, et al. Multisensory convergence in auditory cortex. I. Cortical connections of the caudal superior temporal plane in macaque monkeys. 7 Comp Neurol 2007; 502: 894-923.

10. Robinson DL, Petersen SE. The pulvinar and visual salience. Trends Neurosci 1992; 4: 127-32.

11. Olshausen BA, Anderson CH, Van Essen DC. A neurobiological model of visual attention and invariant pattern recognition based on dynamic routing of information. 7 Neurosci 1993; 13: 4700-19.

12. Guillery RW. Anatomical evidence concerning the role of the thalamus in corticocortical communication. F Anat 1995; 187: 583-92.

13. Guillery RW. Sherman MS thalamic relay functions and their role in corticocortical communication: generalizations from the visual system. Neuron 2002; 33: 1-20.

14. Guillery RW. Anatomical pathways that link action to perception. Prog Brain Res 2005; 149: 235-56.

15. Brodmann K. Localization in the Cerebral Cortex: The Principles of Comparative Localisation in the Cerebral Cortex Based on 
Cytoarchitectonics (transl. with editorial notes and an introduction by Garey LJ). Berlin: Springer; 2005.

16. Mountcastle VB. An organizing principle for cerebral function: the unit module and the distributed system. In: Edelman GM, Mouncastle, VB, editors. The Mindful Brain. Cambridge MA: MIT Press; 1978. p. 7-50.

17. Douglas RJ, Martin KA. Neuronal circuits of the neocortex. Annu Rev Neurosci 2004; 27: 419-51.

18. Harting JK, Updyke BV, Van Lieshout DP. Corticotectal projections in the cat: Anterograde transport studies of twentyfive cortical areas. 7 Comp. Neurol 1992; 324: 379-414.

19. Guillery RW. Branching thalamic afferents link action and perception. 7 Neurophsyiol 2003; 90: 539-48.

20. Taube JS. The head direction signal: origins and sensory-motor integration. Annu Rev Neurosci 2007; 30: 181-207.

21. Guillery RW. Is postnatal neocortical maturation hierarchical? Trends Neurosci 2005; 28: 512-517.
22. Warren RM, Warren RP. Helmholtz on Perception: Its Physiology and Development. New York: Wiley; 1968.

23. Merleau-Ponti M. Phenomenologie de la Perception, 1945 (transl. by Smith C.) London: Routledge; 2002.

24. Noë A. Action in Perception. Cambridge MA: MIT Press; 2004.

25. Noë A. Beyond the eye. Times Literary Supplement 2006; May 6th; $12-13$.

26. Churchland PS, Ramcharan VS, Sejnowski TJ. A critique of pure vision. In: Koch C, Davis JL, eds. Large Scale Neuronal Theories of the Brain. Cambridge MA: MIT Press; 1194. p. 23-65.

27. Berthoz A. The Brain's Sense of Movements. Cambridge Mass: Harvard Universty Press; 2000.

28. Gibson JJ. The Ecological Approach to Visual Perception. Hillsdale, NJ: L Erlbaum; 1979.

29. Varela FJ, Thompson E, Rosch E. The Embodied Mind. London: MIT Press; 1993.

Correspondence to: Ray W. Guillery, MD

Department of Anatomy, Marmara University

Haydarpaşa, Istanbul, Turkey

e-mail: rguiller@facstaff.wisc.edu

Conflict of interest statement: No conflicts declared. 